\title{
Effect of thermal convection on frequency response of a perturbed vaporizing pastille-shaped droplet
}

\author{
Kwassi Anani ${ }^{1, a}$, Roger Prud'homme ${ }^{1, b}$, SÉnA Amah D'Almeida ${ }^{1}$ \\ and Kofi Seylom Assiamoua ${ }^{1}$ \\ Doctoral School of Mathematics and Applications, Department of Mathematics, University of Lomé, BP 1515, Lomé, Togo
}

Received 7 September 2010, Accepted 14 April 2011

\begin{abstract}
We study the dynamic response to small acoustic oscillations of a vaporizing droplet in shape of a pastille (a small liquid cylinder, called "pastille" in the sequel, the height of which being smaller than the radius of the base). Contrary to some previously proposed models, where the thermal convection effect inside the droplet is often neglected, the continuously fed pastille-shaped model takes into account the effects of both thermal convection and conduction. Curves related to different heat exchange coefficients are presented for the frequency response of the vaporization rate. The case where the feeding process at the bottom of the pastille is assumed isothermal (isothermal bottom regime) is compared to the one where the feeding process at the bottom of the pastille is adiabatic (adiabatic bottom regime). The response factor curves for the pure conduction model of the spherical droplet and for the present model of the "equivalent pastille" are also compared. The temperature field perturbation is then examined. As well as for the evaporation mass flow rate perturbation, comparisons are made between the regime with an isothermal bottom and the one with an adiabatic bottom. We find that, in spite of some divergences observed between the various cases, the frequency response of a droplet submitted to acoustic oscillations presents also some common points. It is shown that the life time (or residence time), the thermal diffusion time, and the period of the harmonic perturbation do intervene strongly in the behaviour of the vaporizing pastille. The liquid propulsion is a possible application of this basic study conducted as part of a thesis.
\end{abstract}

Key words: Pastille / evaporation / harmonic oscillations / response factor / temperature perturbation

\section{Introduction}

Much research in combustion has been focused on instability mechanisms. With an emphasis on low frequency oscillations, recent works have been focused on the liquid droplet injected into a hot gaseous environment. This type of instability is also observed in liquid propellant engines where it is coupled by combustion instabilities. The subsequent vaporization and combustion dynamics can contribute to high frequency instability of the whole chamber [1]. Many of these studies about this type of instability have been published. Let us cite Bhatia and Sirignano [2], Culick and Yang [3],

\footnotetext{
${ }^{a}$ Corresponding author: ananikwassi@yahoo.fr

b Emeritus Research Director, Jean Le Rond d'Alembert Institute, UMR 7190, Pierre et Marie Curie University/Centre National de la Recherche Scientifique, Box 162, 4 place Jussieu, 75252 Paris Cedex 05, France
}

Delplanque and Sirignano [4], DiCicco and Buckmaster [5], Dubois et al. [6], Duvur et al. [7], Harrje and Reardon [8], Heidmann and Wieber [9], Heidmann [10], Prud'homme [11,12], Prud'homme et al. $[1,13]$. In all these published documents the authors have developed or refined different theories to better understand the phenomenon.

In general, the dynamic response to oscillations is computed by using drop-evaporation theory [14], on the basis of the Rayleigh criterion [15], with simplifying assumptions $[1,9,10]$. In the Heidmann analogy [10], a spherical droplet of constant volume represents a mean droplet at a fixed place in a chamber, in the steady regime. More precisely, the system frequency response of the spray of repetitively injected drops in the combustion chamber is obtained by considering a mean evaporating droplet at rest, continuously fed at a stationary flow rate. This evaporating droplet represents a mean droplet with 


\section{Nomenclature}

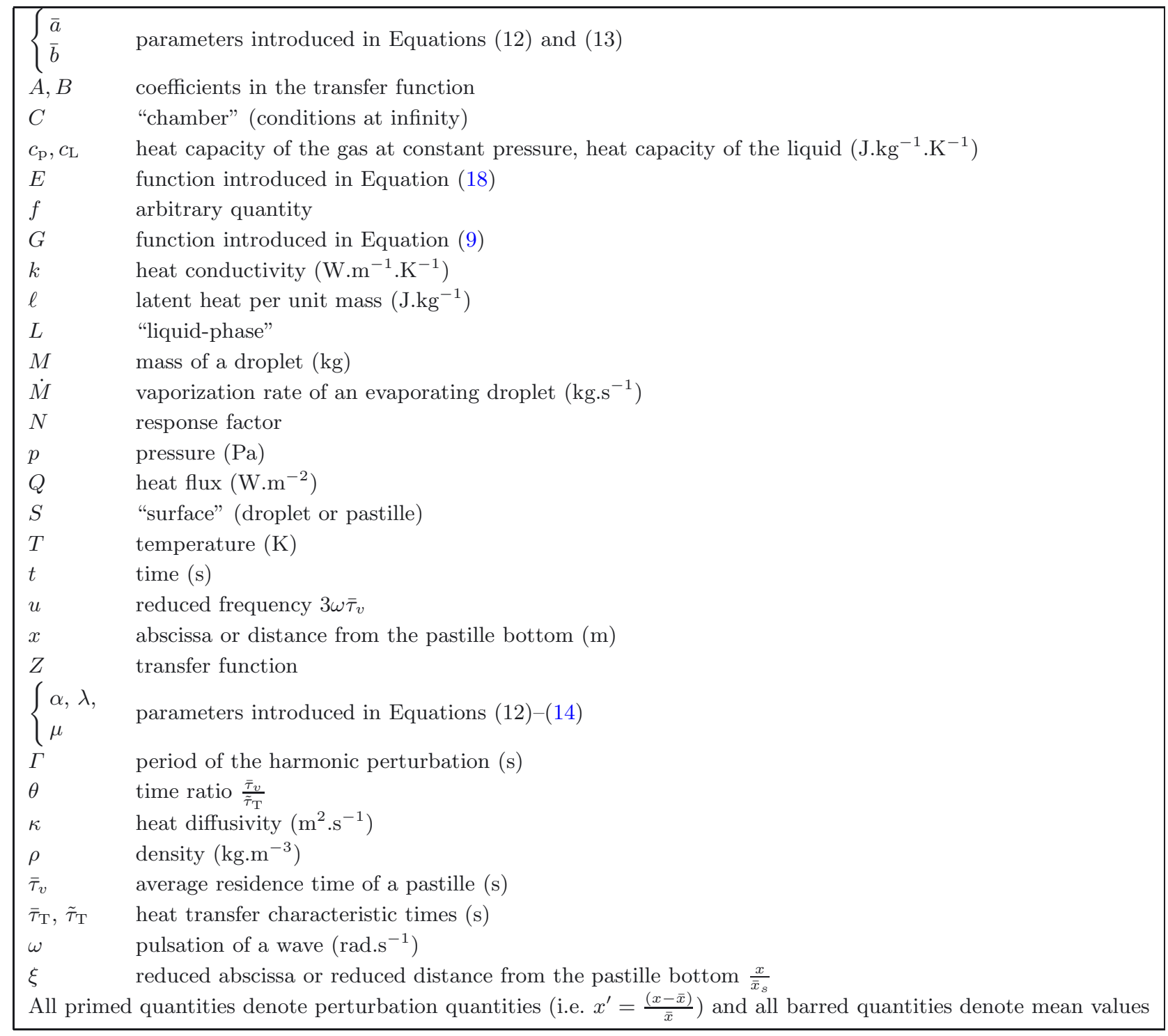

constant volume, at a specified location in the combustion chamber, and is supposed to summarize the frequency response of individual drops in the spray. The droplet is assumed to be fed by fuel at a constant average temperature with a constant injection rate. With the simplification of Heidmann and Wieber [9], an infinite thermal diffusivity of the liquid is considered; therefore the mean droplet has a uniform temperature. In the analysis of Prud'homme et al. [1,13], a finite thermal diffusivity of the liquid is taken into account. The feeding comes from a point source placed at the mean droplet centre. A linearized investigation is performed in which the response factor is considered. This investigation leads to a linear partial differential equation including a convection term with a variable coefficient. The study of the obtained equation is still somewhat complicated except for the pure conduction case. In fact, the linear analysis of the behaviour of a continuously fed spherical droplet subject to small acoustic perturbations has been possible under the following simplifying assumptions:

1. neglected convection effect in the energy conservation equation for the liquid-phase;

2. zero temperature gradient in the centre of the droplet (adiabatic condition).

Out of those cases, no analytical solution has been found and any asymptotical or numerical study has been performed.

The present modelling of the droplet by a cylindrical pastille with an impermeable and adiabatic lateral surface allows us to study analytically the response of the droplet under different other assumptions. The mean evaporating spherical droplet is replaced by an equivalent cylindrical pastille with the same volume and the same evaporating surface. This model leads to a linear partial differential equation with constant coefficients even if the convection 
term is taken into account. It allows to study analytically the convection effect inside the pastille and to compare the behaviour of the system between the isothermal and adiabatic bottom regimes. The latter regimes replace the regimes in the centre of the droplet. Some results of the pastille model are certainly applicable to the spherical droplet model although their geometrical configurations are different. The liquid propulsion is a possible application of the reported basic study. The theoretical results of the present study can also be applied to model the transient combustion control in resonant systems since combustion instabilities still remain to be a formidable challenge for plane and car engine designers.

The main objective of the present study, based on the linear analysis of small harmonic perturbations, is to extend the results from $[1,12]$ to the case where heat convective flow effect is considered during the process. As in $[1,12]$ we will consider the case of a stabilized velocity and look for the response factor, defined as the ratio of evaporating mass flow rate perturbation to the pressure perturbation. We will also be concerned only with vaporization dynamics. The influence of combustion will be limited to imposing a stationary composition and temperature at infinity. The combined effects of vaporization dynamics and combustion kinetics, and their eventual retroaction on ambient pressure will not be analyzed here. We will assume also that the gas-phase is in the quasi-steady regime as in [16-18]. Consequently the derived perturbation equations established for the gas-phase of the evaporating droplet in $[1,12,13]$ will be used for the equivalent pastille.

First, a frequency response of the vaporization process to small oscillations in pressure will be evaluated for the isothermal and adiabatic bottom regimes. Variations in the response factor with frequency and heat thermal exchanged coefficient will be presented for both regimes. In the adiabatic bottom case for the equivalent pastille, comparisons will also be made with the pure conduction model of the spherical droplet. Finally, a specific analysis of temperature field perturbation will be performed. It is shown that, in some concrete cases, significant differences appear between the perturbation propagation for the isothermal and adiabatic bottom regimes. Note that in the rest of the study, the term convection will refer to the radial thermal convection inside the continuously fed droplet from its centre to its evaporation surface or to the axial thermal convection inside the continuously fed pastille from its bottom up to its evaporation surface.

\section{Formulation of the model}

In order to assess the influence of the thermal wave inside the droplet on the unsteady evaporation mechanism, we present an alternative to the more classical model of a spherical droplet. The situation considered here is an idealized model of a motionless, cold pastille after it is placed in a hot stagnant, gravity-free environment of infinite extent. The pastille with an impermeable and adiabatic lateral surface is assumed to be continuously fed at

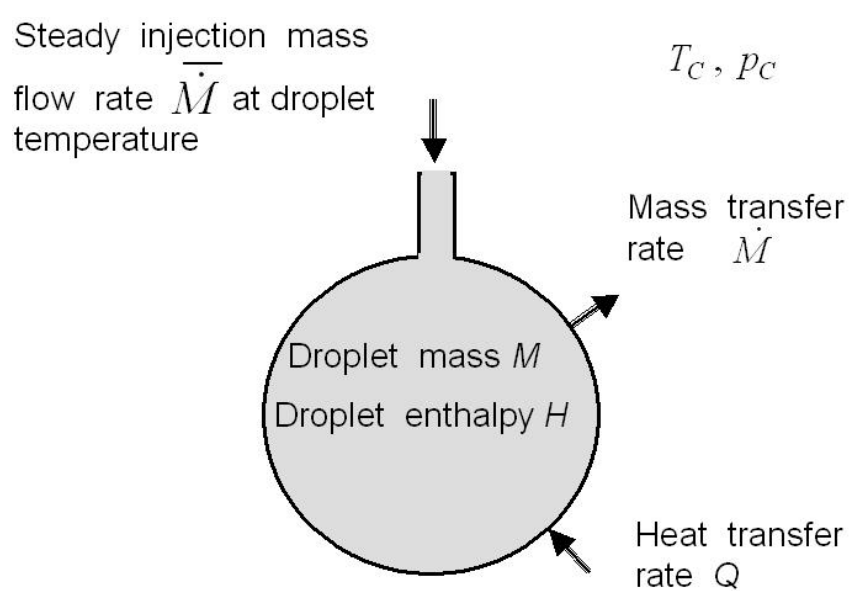

Fig. 1. Droplet of radius $\bar{r}_{S}$, continuously supplied by a steady injection mass flow rate $\overline{\dot{M}}$ of the liquid fuel.

its bottom and is subject to small acoustics oscillations. The system pressure is much less than the critical pressure of the liquid, and therefore critical phenomena are not important.

\subsection{Geometry considerations}

The classical model of a continuously fed spherical droplet was first formulated in 1960s by Heidmann ([10] and references therein). Next, in a simplified approach, Heidmann and Wieber [9] considered a mean evaporating droplet with uniform but time-varying temperature since an infinite thermal diffusivity in the liquid-phase is assumed. Figure 1 illustrates a Heidmann droplet. This evaporating droplet has a constant average radius, and is continuously fed by a steady flow.

The classical model described above was reviewed and substantially refined by Prud'homme et al. [1]. Their analysis, which takes into account a finite thermal diffusivity of the liquid, was based on the assumptions mentioned in the Introduction. Namely, it was assumed that the radial convection effect in the energy equation due to the feeding process at the centre of the droplet is neglected and the droplet centre remains adiabatic. Explicit expression was then derived for the droplet mass transfer function in the pure conduction case and the results were discussed. Also in this study, a numerical analysis of heat transfer inside the mean evaporating droplet, termed the "multilayer model", was reported.

In the present model, the spherical droplet is replaced by a cylindrical pastille-shaped droplet (which will be called "pastille" in the sequel) with mean height $\bar{x}_{S}$ and constant orthogonal section $S$ (Fig. 2). Its volume and evaporation surface are supposed to be equal to those of the droplet. This double condition implies that

$$
\bar{x}_{S}=\frac{\bar{r}_{S}}{3}, \quad r_{\mathrm{C}}=2 \bar{r}_{S}=6 \bar{x}_{S}
$$

with $S=4 \pi \bar{r}_{S}^{2}=\pi r_{\mathrm{C}}^{2}$ and $S \bar{x}_{S}=\frac{4}{3} \pi \bar{r}_{S}^{3}$, and $r_{\mathrm{C}}$ being the radius of the cylindrical pastille. The pastille can 


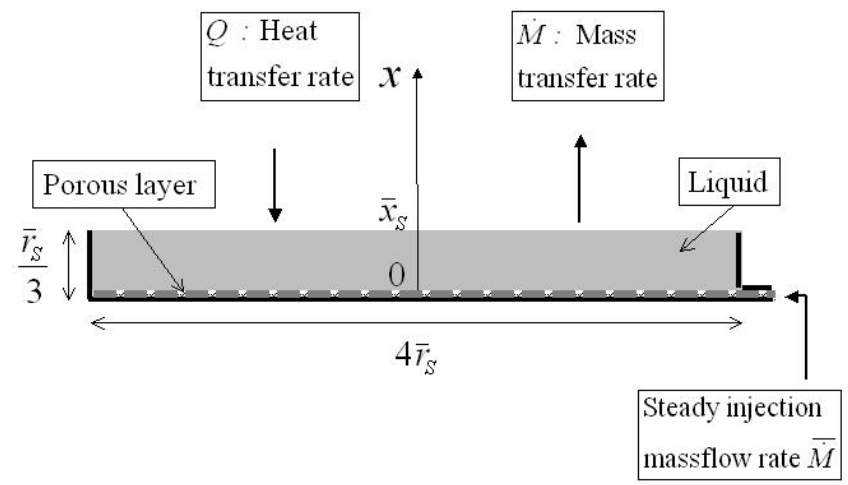

Fig. 2. The equivalent pastille.

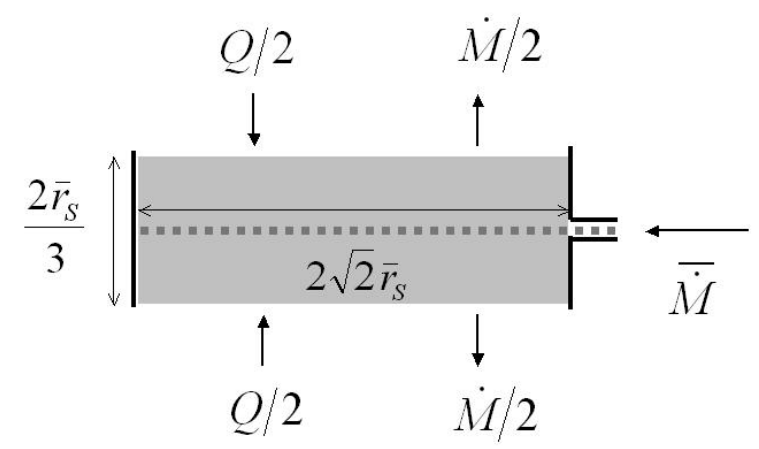

Fig. 3. Another equivalent pastille.

be fed by fuel at the average temperature $\bar{T}_{S}$, with the average mass flow rate $\overline{\dot{M}}$. The feeding is made under the pastille bottom across a flat porous cylinder with the same diameter as the pastille, which permits to assure a uniform flow at $x=0$. The velocity $v_{\mathrm{L}}$ in the liquid-phase is constant and parallel to the axis of the pastille. During the injection, the bottom of the pastille is assumed to be adiabatic (zero temperature gradient) or isothermal (imposed constant temperature).

One may obtain another system equivalent to the continuously fed spherical droplet with two evaporating surfaces (Fig. 3). The pastille is twice as thick and its radius is $\sqrt{2}$ times smaller, and is to be fed this time in its central plane. This more compact system shows the same behaviour as the previous one. The pastille model may be justified according to the small size of the droplet compared to the dimensions of the combustion chamber imposing the perturbations of the pressure $p_{\mathrm{C}}$.

\subsection{The characteristic times}

It has been shown in previous studies $[19,20]$ that in liquid rocket engines, the acoustic periods of the chamber modes (about $10^{-4}$ to $10^{-3} \mathrm{~s}$ ) may be of the same order of magnitude as the characteristic times of vaporization and combustion, whereas the primary and secondary atomization phenomena intervene at smaller time scales. We denote the density, the specific heat and the thermal conductivity of the pastille respectively by $\rho_{\mathrm{L}}, c_{\mathrm{L}}$, and
$k_{\mathrm{L}}$. We assume that these thermal properties remain spatially and temporally constant in the pastille during the process. In this situation two characteristic times intervene. The residence time of the pastille, which replaces the notion of the pastille life time in the present situation of constant volume, is written as $\bar{\tau}_{v}=\frac{\bar{M}}{\overline{\dot{M}}}=\frac{\rho_{L} S \bar{x}_{S}}{\rho_{\mathrm{L}} S v_{\mathrm{L}}}$, that is $\bar{\tau}_{v}=\frac{\bar{x}_{S}}{v_{\mathrm{L}}}$. The transfer time by thermal diffusion process is $\tilde{\tau}_{\mathrm{T}}=\frac{\bar{x}_{S}^{2}}{\kappa_{\mathrm{L}}}$ (where $\kappa_{\mathrm{L}}=\frac{k_{\mathrm{L}}}{\rho_{\mathrm{L}} c_{\mathrm{L}}}$ is the thermal diffusivity of the liquid) and is identical to the one of the spherical droplet. Thus we had the same ratio $\theta=\frac{\bar{\tau}_{v}}{\tilde{\tau}_{\mathrm{T}}}$ as for the spherical droplet [1].

One can estimate that the conduction mode will dominate if $\tilde{\tau}_{\mathrm{T}} \ll \bar{\tau}_{v}$. On the contrary, for $\tilde{\tau}_{\mathrm{T}} \gg \bar{\tau}_{v}$ the convection of fuel will be dominant. The two modes will coexist for $\tilde{\tau}_{\mathrm{T}} \approx \bar{\tau}_{v}$. As the equivalent pastille with two evaporating surfaces (Fig. 3) is fed this time in its central plane, the characteristic times $\bar{\tau}_{v}$ and $\tilde{\tau}_{\mathrm{T}}$ are unchanged as well as their ratio $\theta$.

The total mass balance of the pastille is

$$
\frac{\mathrm{d} M}{\mathrm{~d} t}=\overline{\dot{M}}-\dot{M}
$$

In a stabilized regime, one has: $\dot{M} \equiv \overline{\dot{M}}, \frac{\mathrm{d} M}{\mathrm{~d} t}=0$ and $M=\bar{M}$.

\subsection{Energy conservation equations}

In the presence of the internal motion, the unsteady heat and mass transfer processes inside the pastille are described by the following system:

$$
\left\{\begin{array}{l}
\frac{\partial T_{l}}{\partial t}+v_{L} \frac{\partial T_{l}}{\partial x}-\kappa_{L} \frac{\partial^{2} T_{l}}{\partial x^{2}}=0 \\
\overline{\dot{M}}=S \rho_{\mathrm{L}} v_{\mathrm{L}}
\end{array}\right.
$$

subject to $T_{l}(0, t)=\bar{T}_{s}(t), T_{l}\left(x_{s}, t\right)=T_{s}(t)$ for the isothermal injection at the bottom or to $\left.\frac{\partial T_{l}}{\partial x}\right|_{0, t}=0$, $T_{l}\left(x_{s}, t\right)=T_{s}(t)$ for the adiabatic injection at the bottom. The flow condition at the pastille surface:

$$
\left.S k_{\mathrm{L}} \frac{\partial T_{l}}{\partial x}\right|_{x_{s}, t}=Q_{L}=Q-\dot{M} \ell
$$

couples the gas and the liquid-phase solutions.

\section{Linear analysis for small perturbations}

In the vaporization calculations, the acoustic oscillations affect the pastille heat and mass transfer processes, causing perturbations in the pastille temperature and in its mass evaporation rate. With these fluctuations, the rate of vaporization depends on the frequency of the oscillation. The transfer function of the frequency response can be evaluated. 


\subsection{The linearized equations}

Assuming that the pastille has reached a steady regime, we now consider small acoustic perturbations writing $\Delta f=f-\bar{f}$ where $f$ is a flow parameter, $\bar{f}$ is its mean value, $\Delta f$ is the corresponding absolute perturbation, and $f^{\prime}=\frac{\Delta f}{f}$ is the corresponding relative perturbation. The energy conservation equation becomes

$$
\frac{\partial T_{l}^{\prime}}{\partial t}+v_{\mathrm{L}} \frac{\partial T_{l}^{\prime}}{\partial x}-\kappa_{\mathrm{L}} \frac{\partial^{2} T_{l}^{\prime}}{\partial x^{2}}=0
$$

with $v_{\mathrm{L}}=\frac{\overline{\dot{M}}}{\pi \bar{r}_{\mathrm{c}}^{2} \rho_{\mathrm{L}}}$, and the flow at the surface is given by

$$
\left.\pi \bar{r}_{\mathrm{c}}^{2} k_{\mathrm{L}} \bar{T}_{S} \frac{\partial T_{l}^{\prime}}{\partial x}\right|_{\bar{x}_{S}, t}=\Delta Q_{\mathrm{L}}
$$

The boundary conditions in the isothermal bottom regime become

$$
\left\{\begin{array}{l}
T_{l}^{\prime}(0, t)=\bar{T}_{S}^{\prime}=0 \\
T_{l}^{\prime}\left(\bar{x}_{s}, t\right)=T_{S}^{\prime}(t)
\end{array}\right.
$$

and in the adiabatic bottom regime we set

$$
\left\{\begin{array}{c}
\left.\frac{\partial T_{l}^{\prime}}{\partial x}\right|_{0, t}=0 \\
T_{l}^{\prime}\left(\bar{x}_{s}, t\right)=T_{S}^{\prime}(t)
\end{array}\right.
$$

\subsection{Analytical solutions}

The left hand side of Equation (2) consists of three terms, representing change in time, convection, and diffusion. Introducing harmonic perturbations of the form $f^{\prime}=$ $\hat{f}(x) \mathrm{e}^{i \omega t}$, we set $T_{l}^{\prime}=\hat{T}_{l} \mathrm{e}^{i \omega t}$ and $\Delta Q_{L}=\Delta \hat{Q}_{L} \mathrm{e}^{i \omega t}$. We find a solution in the form:

$$
\hat{T}_{l}=C\left(\mathrm{e}^{s^{+} x}-\mathrm{e}^{s^{-} x}\right)
$$

with $C$ being a constant, $s^{+}, s^{-}$are two complex roots of the following characteristic equation:

$$
i \omega+\frac{s \bar{x}_{S}}{\bar{\tau}_{v}}-\frac{\left(s \bar{x}_{S}\right)^{2}}{\tilde{\tau}_{\mathrm{T}}}=0
$$

The flow condition at the surface is

$$
\left.S k_{\mathrm{L}} \bar{T}_{S} \frac{\mathrm{d} \hat{T}_{l}}{\mathrm{~d} x}\right|_{\bar{x}_{s}, t}=\Delta \hat{Q}_{\mathrm{L}}
$$

Let us introduce the reduced frequency $u=3 \omega \bar{\tau}_{v}$, the same expression as in the spherical droplet case [1]. The flow condition at the surface leads to

$$
\Delta \hat{Q}_{\mathrm{L}}=\frac{S k_{\mathrm{L}} \bar{T}_{S}}{\bar{x}_{S}} G(u, \theta) \hat{T}_{S} .
$$

For the isothermal bottom case, using conditions (4), one has $C=\frac{\hat{T}_{S}}{e^{s+\bar{x}_{S}-e^{s^{-} \bar{x}_{S}}}}$ and

$$
G(u, \theta)=\frac{s^{+} \bar{x}_{S} \mathrm{e}^{s^{+} \bar{x}_{S}}-s^{-} \bar{x}_{S} \mathrm{e}^{s^{-} \bar{x}_{S}}}{\mathrm{e}^{s^{+} \bar{x}_{S}}-\mathrm{e}^{s^{-} \bar{x}_{S}}}
$$

For the adiabatic bottom case, conditions (5) provide

$$
C=\frac{\hat{T}_{S}}{\frac{\mathrm{e}^{s^{+} \bar{x}_{S}}}{s^{+} \bar{x}_{S}}-\frac{\mathrm{e}^{s^{-} \bar{x}_{S}}}{s^{-} \bar{x}_{S}}}
$$

and

$$
G(u, \theta)=\frac{\mathrm{e}^{s^{+} \bar{x}_{S}}-\mathrm{e}^{s^{-} \bar{x}_{S}}}{\frac{\mathrm{e}^{s^{+} \bar{x}_{S}}}{s^{+} \bar{x}_{S}}-\frac{\mathrm{e}^{s^{-} \bar{x}_{S}}}{s^{-} \bar{x}_{S}}}
$$

\subsection{The transfer function}

We will now apply to the evaporation surface of the cylindrical drop the same fields of temperature and pressure than those obtained with the theory of quasistationary spherical drop $[1,11]$. The ambient pressure is given by $p^{\prime}=\hat{p}_{C} e^{i \omega t}$. From the study of the gas phase it can be deduced that:

$$
\hat{\dot{M}}=\alpha \frac{i u}{1+i u}\left(\bar{b} \hat{T}_{S}-\hat{p}_{C}\right)
$$

with $u=3 \omega \bar{\tau}_{v}, \dot{M}^{\prime}=\frac{\Delta \dot{M}}{\dot{M}}=\hat{\dot{M}} \mathrm{e}^{i \omega t}$, and

$$
\Delta \hat{Q}_{\mathrm{L}}=\overline{\dot{M}} \bar{\ell}\left(\bar{a} \hat{p}_{C}-\mu \hat{T}_{S}\right)
$$

with $\Delta Q_{\mathrm{L}}=Q_{\mathrm{L}}-Q_{\text {stat }}=Q_{L}=\Delta \hat{Q}_{\mathrm{L}} \mathrm{e}^{i \omega t}$. Eliminating $\Delta \hat{Q}_{\mathrm{L}}$ in (13) and (9), the expression for the complex transfer function $Z=\frac{1}{\alpha} \frac{\hat{\dot{M}}}{\hat{p}_{C}}$ is obtained:

$$
Z(u, \theta)=\frac{i u}{1+i u} \frac{A-3 \theta G(u, \theta)}{B+3 \theta G(u, \theta)}
$$

with $A=\frac{3(\bar{a} \bar{b}-\mu)}{\lambda}, B=\frac{3 \mu}{\lambda}$ (the parameters $\bar{a}, \bar{b}, \mu$ and $\alpha$ are the same as defined in [1]). To obtain the expression of the function $G$, it is necessary to solve the characteristic Equation (7). We set $i \frac{u}{3 \theta}+\frac{1}{4 \theta^{2}}=R \mathrm{e}^{i \varphi}, d=\tan \left(\frac{\varphi}{2}\right)$ with $R$ being the modulus of $i \frac{u}{3 \theta}+\frac{1}{4 \theta^{2}}$ which is $R=$ $\sqrt{\left(\frac{1}{4 \theta^{2}}\right)^{2}+\left(\frac{u}{3 \theta}\right)^{2}}$ and get $d$ as a function of $u$ and $\theta$ :

$$
d=-\frac{3}{4 \theta u}+\sqrt{1+\left(\frac{3}{4 \theta u}\right)^{2}}
$$

Thus, we find the two solutions $s^{+}$and $s^{-}$of Equation (7). We now set $m=\frac{1}{2 \theta}+\sqrt{R} \mathrm{e}^{i \frac{\varphi}{2}}$ and $n=\frac{1}{2 \theta}-\sqrt{R} \mathrm{e}^{i \frac{\varphi}{2}}$. Using respectively the expressions (10) and (11) we obtain for the isothermal bottom regime:

$$
G=\frac{m \mathrm{e}^{m}-n \mathrm{e}^{n}}{\mathrm{e}^{m}-\mathrm{e}^{n}}
$$


and for the adiabatic bottom regime:

$$
G=\frac{\mathrm{e}^{m}-\mathrm{e}^{n}}{\frac{\mathrm{e}^{m}}{m}-\frac{\mathrm{e}^{n}}{n}}
$$

If we neglect the convection heat transfer effect in the pastille and consider simply a pure conduction circumstance, the characteristic equation takes the form $i \omega-$ $\frac{\bar{x}_{S}^{2} s^{2}}{\tilde{\tau}_{T}}=0$ and the expression of $G$ in the isothermal bottom case appears as

$$
G(u, \theta)=(1+i) \sqrt{\frac{u}{6 \theta}} \operatorname{coth}\left[(1+i) \sqrt{\frac{u}{6 \theta}}\right]
$$

For the adiabatic bottom case, $G$ is given by

$$
G(u, \theta)=\frac{(1+i) \sqrt{\frac{u}{6 \theta}}}{\operatorname{coth}\left[(1+i) \sqrt{\frac{u}{6 \theta}}\right]}
$$

\section{Response factor}

A sinusoidal oscillation in pressure causes an oscillation in vaporization rate that depends on the same frequency. The response is the resulting heat or mass perturbation. We analyze in this section the response of the vaporization rate of the pastille to an acoustic oscillation.

\subsection{The reduced response factor}

The reduced pressure perturbation is defined as $p^{\prime}=$ $\frac{(p-\bar{p})}{\bar{p}}$, and the resulting reduced heat or mass perturbation is $q^{\prime}=\frac{(q-\bar{q})}{\bar{q}}$. The response factor $N$ is defined as

$$
N=\frac{\iint_{V, t} q^{\prime}(V, t) p^{\prime}(V, t) \mathrm{d} t \mathrm{~d} V}{\iint_{V, t}\left(p^{\prime}(V, t)\right)^{2} \mathrm{~d} t \mathrm{~d} V}
$$

For sinusoidal oscillations which are uniform over a finite volume: $N=\frac{|\hat{q}|}{|\hat{p}|} \cos \phi$, where $|\hat{q}|,|\hat{p}|$ are the moduli and $\phi$ is the phase difference between $q^{\prime}$ and $p^{\prime}$. The response factor $N$ is expressed as the ratio of the magnitude of heat or mass perturbation to the magnitude of the pressure perturbation and thus, includes phase relations. In the rest of this paper we will consider and call "response factor", the reduced response factor, which is the real part of the transfer function $Z[11]$ :

$$
\frac{N}{\alpha}=\Re(Z)
$$

The response factor is assumed positive when the vaporization rate and the chamber pressure are either above or below their mean values and assumed negative when the vaporization rate and the chamber pressure are on the opposite sides of their means. Following the wellknown Rayleigh criterion [15], an unsteady evaporation and burning can be one possible driving mechanism of instability $[8,21]$.

\subsection{Results and discussions}

Our goal is to relate the response factor characteristics to arbitrary changes of the exchange coefficient $\theta$ (ratio of the characteristic times of the process). To draw a response factor curve with the thermal convection transfer effect, it is necessary to replace $\frac{\varphi}{2}$ in the expressions (16) and $(17)$ by $\arctan (d)$ taking into account that $d$ is a function of $u$ and $\theta$ by relation (15). The response factor curves are plotted in (Fig. 4) as functions of the reduced frequency $u$. The variation in the response factor with frequency is defined as the frequency response of the vaporization process. In each diagram of Figure 4, different response factor curves corresponding to particular values of parameter $\theta$ are presented. With or without thermal convection effect, some differences are noticed between the isothermal and adiabatic bottom regimes. All the presented curves have been obtained with $A=10$ and $B=100$. Note that these values of $A$ and $B$ are used by Prud'homme in [12]. They are arbitrary chosen, but they correspond to orders of magnitude of values encountered in the classical fuels. In general, larger negative response factors were observed as the reduced frequency $u$ increases. However, the frequency for a peak or a nonnegative maximum response, if it exists, is relatively unaffected by the variations of $\theta$. A response factor of less than zero will always signify that the evaporation mechanism has a stabilizing influence upon the system. The cut-off reduced frequency $u_{c}$ for a zero response factor, therefore, divides the frequency response into regions of destabilizing and stabilizing influences, and may be considered as a critical frequency.

In the isothermal bottom regime, a positive response region and therefore a critical frequency $u_{c}$ exist for low values of the thermal exchange coefficient $(1 \leq \theta<4)$. When $\theta$ increases, $u_{c}$ and also the $u$ value at the positive maximum response, decrease quickly towards 0 . The decrease is accelerated when the thermal convection affects the system. With or without thermal convection effect, the curves of response factor are completely under the $X$ axis for large $\theta$ values. The absence of region of positive response factor for increasing values of $\theta$ may cause more stability in the combustion chamber. We conclude that in this regime, the convection affects significantly the peak value of the response factor at low $\theta$ values $(1 \leq \theta<4)$ and has also an appreciable decreasing effect on the critical frequency $u_{c}$. Positive intervals for response factor curves, if they exist, are then affected by the convection as shown in Figure $4 \mathrm{a}$ and Figure $4 \mathrm{c}$ for $\theta=1,2$.

In the adiabatic bottom regime, the response factor exhibits a peak value at a specific frequency. It approaches zero at a lower frequency and decreases to negative values at higher frequencies. When $\theta$ increases from 1 on, taking into account the thermal convection effect or not, the critical frequency $u_{c}$ tends to decrease quickly first, reaches a minimum value about $\theta=4$ and then begins to increase very slowly and tends to attain a limit frequency value slightly greater than 30 . On the other hand, the peak value increases slightly with $\theta$ and tends to a value about 0.1. As shown in Figures $4 \mathrm{~b}$ and $\mathrm{d}$, the critical 


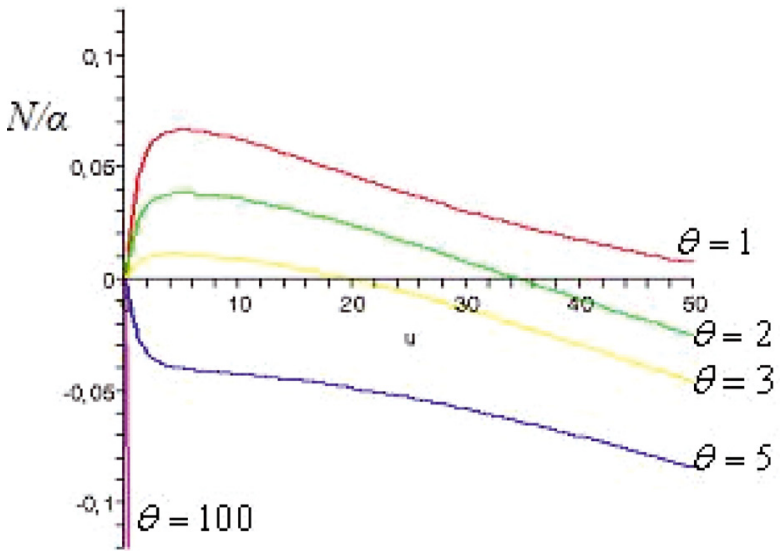

(a)

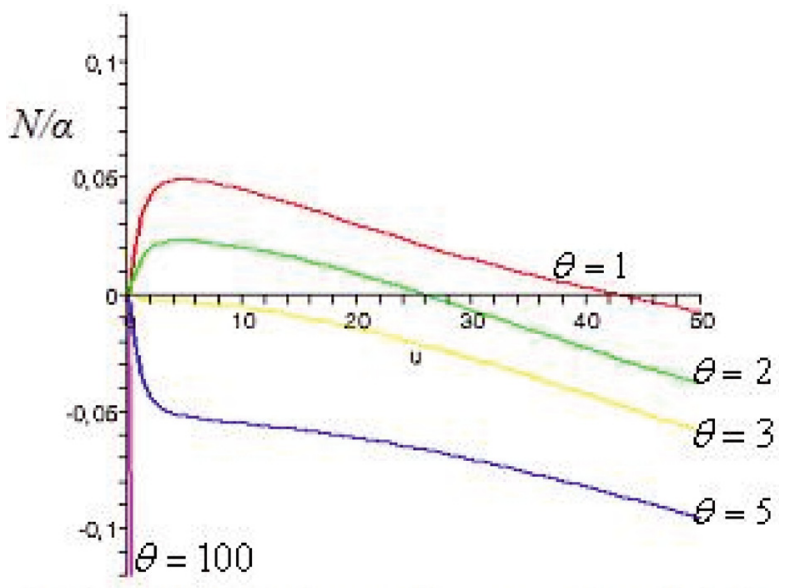

(c)

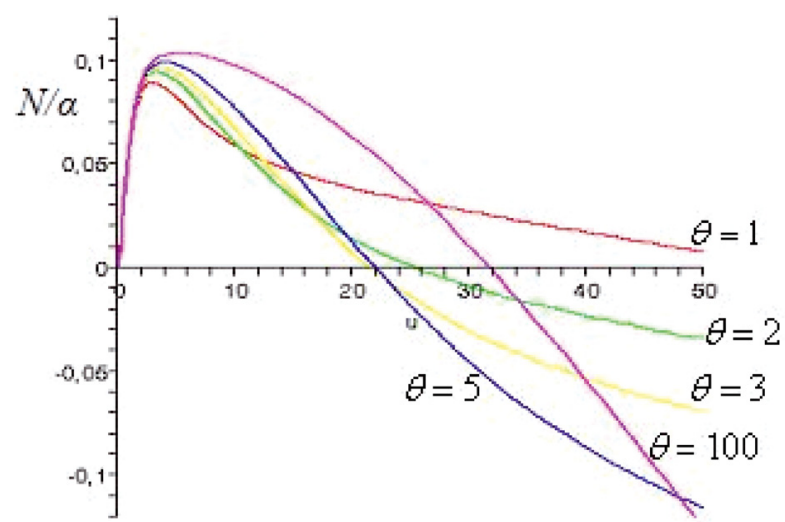

(b)

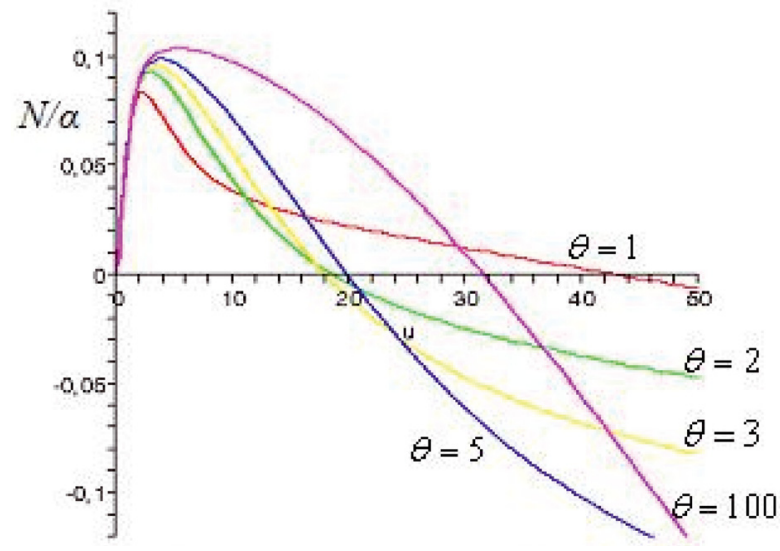

(d)

Fig. 4. Influence of reduced exchange coefficient $\theta$ on the response factor for the pastille with $A=10$ and $B=100$. (a) Isothermal bottom without convection effect. (b) Adiabatic bottom without convection effect. (c) Isothermal bottom with convection effect. (d) Adiabatic bottom with convection effect.

frequencies $u_{c}$ (corresponding to $N=0$ ) are slightly smaller for the cases of response factor in the presence of the convective effect in the system. One concludes that, in this regime, when we assume $\theta \geq 1$, the thermal convection effect reduces slightly the critical frequency value and has only a little effect on the shape of the response curve as is shown in Figures $4 \mathrm{~b}$ and $\mathrm{d}$.

Comparisons with the pure conduction model for the spherical droplet are valid only within the context of an adiabatic bottom regime. Assuming that the thermal convection effect is neglected, the study of the transfer function for the spherical droplet with a finite conductivity gives (see $[1,11])$ :

$$
Z(u, \theta)=\frac{i u}{1+i u} \frac{A+\theta E(u, \theta)}{B-\theta E(u, \theta)}
$$

where $E=1-s_{0} r_{S} \operatorname{coth}\left(s_{0} r_{S}\right)$ with $s_{0} r_{S}=(1+i) \sqrt{\frac{3 u}{2 \theta}}$, $\theta=\frac{9 \kappa_{L} \bar{\tau}_{v}}{\bar{r}_{S}^{2}}=\frac{9 \bar{\tau}_{v}}{\bar{\tau}_{\mathrm{T}}}$ and $\bar{\tau}_{\mathrm{T}}=\frac{\bar{r}_{S}^{2}}{\kappa_{L}}$. The influence of the reduced exchange coefficient $\theta$ on the response factor
$\frac{N}{\alpha}=\Re(Z)$ is shown in Figure 5. Assuming also the absence of the thermal convection effect in the equivalent vaporizing pastille model, we notice that response factor curves for the pastille Figure $4 \mathrm{~b}$ and the equivalent droplet Figure 5 present several similarities with respect to their shapes, for an arbitrary given value of $\theta$. However, we also notice, for low values of $\theta(1<\theta<4)$, a reduction of the critical frequency leading to a reduction of the positive region for the vaporizing pastille model. The phenomenon is slightly reversed for the large values of $\theta$ but then the critical frequencies $u_{c}$ for both models tend to a constant value about 30. Assuming now a convective effect uniquely for the vaporizing pastille model, we see that the curves for the pastille (Fig. 4d) and the equivalent droplet (Fig. 5) have the same appearance for $\theta>1$. As for the foregoing comparison, we note here for $1<\theta<4$ a critical frequency displacement, but no appreciable change occurs in the shapes of the curves for the conditions examined.

To conclude, we note that for $\theta \geq 2$ the curves of the two models of pastille (with or without thermal 


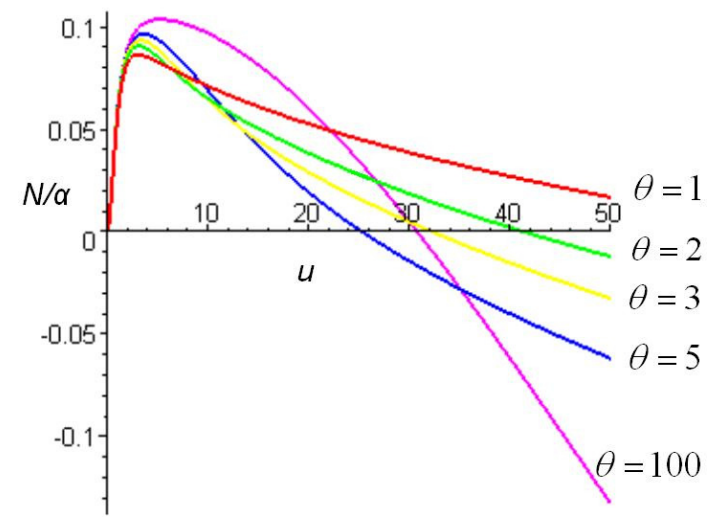

Fig. 5. Influence of reduced exchange coefficient $\theta$ on the response factor for the spherical droplet with an adiabatic condition at its centre with $A=10$ and $B=100$.

convection effect) are similar but differ from those in the pure conduction case by a light reduction of positive region (instability domain). A complete response similarity for a pure conduction droplet model and an equivalent pastille model occurs uniquely for very large values of $\theta \gg 1$. We can see that neglecting the thermal convection effect in droplet model gives correct results for sufficiently large theta. For the continuously fed spherical droplet model one may then neglect the convective term for large values of $\theta(\theta \gg 1)$.

\subsection{Analysis of results}

A first important remark concerning the diagrams of (Fig. 4) is that peak values of the response factor for both adiabatic and isothermal bottom regimes occur at the same reduced frequency of about $u=3$, that is $u=3 \omega \bar{\tau}_{v} \cong 3$ or $\omega \bar{\tau}_{v} \cong 1$. This means that the response factor is nearly at maximum when the entire pastille lifetime (residence time $\bar{\tau}_{v}$ ) equals the oscillation period. As the pastille lifetime approaches the period of oscillation, the pastille temperature can respond to the acoustic oscillations. This favours mass transfer in phase with pressure and results in a peak positive response factor. When the pastille residence time is very small compared to the cycle time, the response factor tends to be zero. In the limit of a very small pastille residence time, the propellant is consumed as fast as it is injected regardless of the combustion chamber conditions. This results in the response factor of zero. The response factor is negative when residence time is large compared to the cycle time, and thus, many oscillations occur in the vaporization process. In this region of negative response, the thermal inertia of the pastille is large relative to the oscillation frequency, and the temperature of the pastille grows from its injection value to the equilibrium temperature with only a small oscillatory component. That is the cause of negative response when the lifetime of the pastille is large.

The value of $\theta$ has also a significant influence on the reduction of the amplification domain (positive region) in both adiabatic and isothermal bottom regimes. In fact, significant differences in the frequency response for the isothermal bottom regime compared to the adiabatic one are smaller peak values of the response factor and the absence of a region of positive response factor for large $\theta$ values. Since $\theta=\frac{\bar{\tau}_{v}}{\tilde{\tau}_{T}}$ is large, that is $\bar{\tau}_{v} \gg \tilde{\tau}_{T}$, the thermal diffusivity characteristic time is less than the pastille life or residence time and the pastille temperature reaches rapidly the equilibrium value. This is especially apparent when $\theta>4$ for curves in Figures $4 \mathrm{a}$ and $\mathrm{c}$ in which the pastille temperature is held constant, since the feeding process at the bottom of the pastille is isothermal. The response factor is negative for all frequencies. This fact is readily confirmed by theoretical consideration since, in the isothermal bottom regime, with or without thermal convection effect, we obtain:

$$
\frac{N}{\alpha} \rightarrow \frac{-u^{2}}{1+u^{2}}
$$

when $\theta \rightarrow \infty$. In the adiabatic bottom regime, the cut-off frequency $u_{c}$, which is a function of $\theta$, tends to a limit value slightly greater than 30 . One may note that in this case the adiabatic injection effect at the bottom tends to counterbalance the diffusion of heat from the pastille surface. Therefore, the boundary conditions relative to the thermal conduction in propellant injection can have a significant effect on the frequency response of vaporization process.

The thermal convection has a negligible effect on the frequency response for $\theta>1$ when the bottom of the pastille is assumed adiabatic. This is predictable since in the linearized Equation (2), the convection term $v_{L} \frac{\partial \mathrm{T}_{l}^{\prime}}{\partial x}$ is proportional to $\frac{1}{\theta} \frac{\partial T_{l}^{\prime}}{\partial x}$. The latter term is close to 0 when $\theta>1$ since $\frac{\partial T_{l}^{\prime}}{\partial x}$ is small for the adiabatic bottom regime. This approximation implies also that for very small values of $\theta(\theta \ll 1)$, the frequency response process may result in the reduction or complete absence of a region of negative response factor and thus, may lead to more instability in the combustion chamber even in isothermal bottom regime. Indeed, assuming the absence of the thermal convection effect in both isothermal and adiabatic bottom regimes, one finds that

$$
\frac{N}{\alpha} \rightarrow \frac{A}{B} \frac{u^{2}}{1+u^{2}}
$$

when $\theta \rightarrow 0$. Assuming now the thermal convection effect in both regimes, we obtain

$$
\frac{N}{\alpha} \rightarrow \frac{16 A-48}{16 B+48} \frac{u^{2}}{1+u^{2}}
$$

when $\theta \rightarrow 0$. In our case $A=10$ and $B=100$ so, for $\theta \ll 1$, the curves of the response factor in both regimes tend respectively to positive values 0.1 and 0.068 when $u \rightarrow \infty$. When $\theta>1$, the thermal convection effect in both adiabatic and isothermal bottom regimes tends to reduce the critical frequency $u_{c}$. In addition, one finds an appreciable reduction of the peak value of the response factor curves in the isothermal bottom case. Therefore 
the convection tends to reduce the positive interval of the response factor curve, and constitute, in that way, an important source of stability in the engine combustion chamber.

In the adiabatic bottom regime, the droplet pure conduction model and the equivalent pastille model without thermal convection effect display very similar behaviour for a given value of $\theta$. When in addition $\theta \gg 1$, behaviour of these two models becomes similar to the one of the equivalent pastille with thermal convection effect. Indeed, for the droplet pure conduction model and the equivalent pastille model with or without thermal convection effect, we have

$$
\frac{N}{\alpha} \rightarrow \frac{(A+B+A B) u^{2}-u^{4}}{B^{2}+\left(1+B^{2}\right) u^{2}+u^{4}}
$$

when $\theta \rightarrow \infty$ and therefore, critical frequencies or cut-off values tend to a constant value equal to $\sqrt{A+A B+B}$ (@33.3 for $A=10$ and $B=100$ ). Accordingly, in the limit of infinitely high values of $\theta$ one has for the equivalent pastille model with or without thermal convection effect the same response factor already found in [1] for the case of the spherical droplet with infinite thermal conductivity. A typical response curve attains a peak value at a specific frequency. It approaches zero at lower frequencies and decreases asymptotically to a negative value equal to -1 at higher frequencies, as we can deduce from the response factor expression in the case of the droplet with infinite thermal conductivity. Critical frequencies are then nearly constant. This behaviour is characteristic of a resonant system, where a peak response occurs at some frequency and a negative feedback occurs at a higher frequency. If this behaviour is realistic, it is significant to the problem of combustion instability in the chamber. It implies that the vaporization process may be tuned to the acoustic frequency of the combustion cavity.

\section{Temperature field}

We assume in this section that convection always affects the system, whether the feeding process at the bottom of the pastille is assumed to be adiabatic or isothermal. As the response factor is the frequency response of mass transfer to the acoustic oscillations, the reduced temperature perturbation is the response of the temperature field to the same acoustic forcing.

\subsection{The reduced temperature perturbation}

We deduce from (12) and (14) the following relation for the value of $\hat{T}_{S}$ :

$$
\hat{T}_{S}=\frac{\hat{\dot{M}}}{Z \alpha \bar{b}} \frac{A-3 \theta G(u, \theta)}{B+3 \theta G(u, \theta)}+\frac{\hat{p}_{C}}{\bar{b}}
$$

The reduced temperature perturbation is defined as

$$
T_{\text {lred }}^{\prime}(u, \theta, \xi, \tau)=\Re\left(\hat{T}_{\text {lred }} \mathrm{e}^{i u \frac{\tau}{3}}\right)
$$

for the reduced quantity $\hat{T}_{\text {lred }}=\frac{\bar{b}}{\hat{p}_{C}} \hat{T}_{l}$ with $\xi=\frac{x}{\bar{x}_{S}}$ and $\tau=\frac{t}{\bar{\tau}_{v}}$.

Taking into account the expression of $\hat{T}_{l}$ in relation (6) and the expression of the transfer function $Z=\frac{1}{\alpha} \frac{\hat{M}}{\hat{p}_{C}}$, we have for the isothermal bottom regime:

$$
T_{\text {lred }}^{\prime}(u, \theta, \xi, \tau)=\Re\left(\frac{(A+B)\left(\mathrm{e}^{m \xi}-\mathrm{e}^{n \xi}\right) \mathrm{e}^{\frac{1}{3} i u \tau}}{(B+3 \theta G)\left(\mathrm{e}^{m}-\mathrm{en}\right)}\right)
$$

with the expression of $G$ corresponding to the isothermal bottom case, i.e. obtained from the relation (10). For the adiabatic bottom regime, the calculation yields:

$$
T_{\text {lred }}^{\prime}(u, \theta, \xi, \tau)=\Re\left(\frac{(A+B)\left(n \mathrm{e}^{m \xi}-m \mathrm{e}^{n \xi}\right) \mathrm{e}^{\frac{1}{3} i u \tau}}{(B+3 \theta G)\left(n \mathrm{e}^{m}-m \mathrm{e}^{n}\right)}\right)
$$

with the expression of $G$ corresponding to the adiabatic bottom case, i.e. obtained from the relation (11).

\subsection{Results and discussions}

Plotting the reduced temperature perturbation $T_{\text {lred }}^{\prime}(u, \theta, \xi, \tau)=\Re\left(\hat{T}_{\text {lred }} \mathrm{e}^{i u \frac{\tau}{3}}\right)$ as function of the reduced abscissa $\xi$ is realized through browsing in time over semi-period $\left(\tau=\frac{t}{\overline{\tau_{v}}}=0, \frac{\pi}{u}, \frac{2 \pi}{u}\right)$ for different values of $\theta$. The cases $\theta=5$ and $u=15,150,1500$ for both isothermal and adiabatic bottom regimes are presented in Figure 6. The penetration depth $\Delta_{l}$ is localized by the abscissa $\xi_{l}$ from which the amplitude of the thermal oscillation becomes null. Legends of the curves in Figure 6 present the variation of the temperature perturbation amplitude related to varying time, $\tau$, and the magnitude of the wave penetration depth inside the pastille. The 3D graph in Figure 7 shows the variations of the reduced temperature perturbation $T_{\text {lred }}^{\prime}$ according to the time $\tau$ and the reduced abscissa $\xi$ at a fixed frequency $u$. All the shown curves have been calculated with $A=10$ and $B=100$.

In the isothermal bottom regime, with reduced frequency at $u=15$ and varying $\theta=(0.1,5,50)$, the amplitude of the thermal oscillation which is zero for $\xi=0$ (bottom of the pastille), increases with $\xi$ and reaches a maximum value at $\xi=1$ (surface of the pastille). Nevertheless, this maximum value at the surface reduces when $\theta$ increases. So, for $\xi=1$ and $\tau=0$, the maximal value of the temperature perturbation at the evaporating surface is approximately $(1,0.8,0.4)$ for the three given values of $\theta=(0.1,5,50)$ respectively. We note also that at a fixed frequency $u$, the greater $\theta$ is, the greater is the penetration depth $\Delta_{l}$ of the thermal wave is. The same phenomenon is observed when the value of $u$ is fixed at 150 and also at 1500 . We nevertheless observe that the increasing of the value of the reduced frequency $u$ at the fixed $\theta$ reduces the penetration depth $\Delta_{l}$ of the wave inside the pastille and also the maximum amplitude of the oscillation at the evaporating pastille surface, as the 3D graph in Figure 7 shows. The phenomenon is clearly expressed 


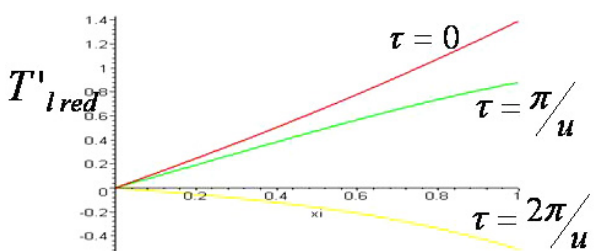

(a)

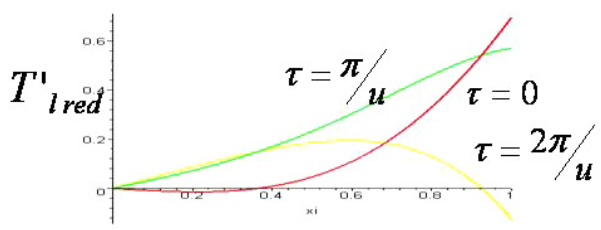

(c)

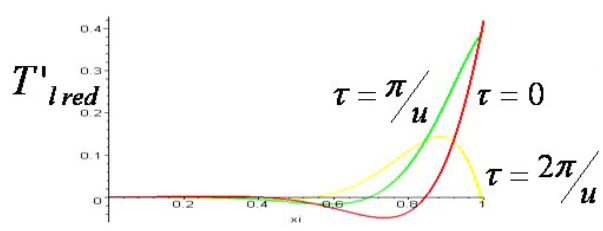

(e)

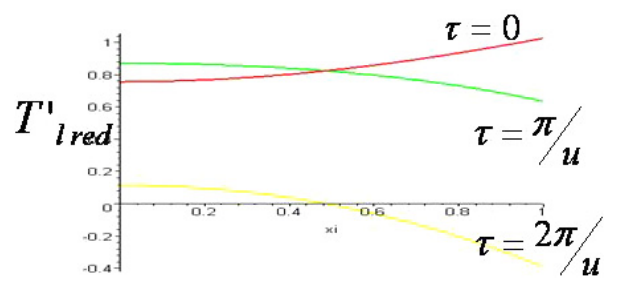

(b)

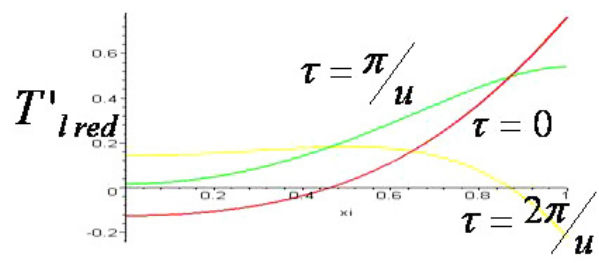

(d)

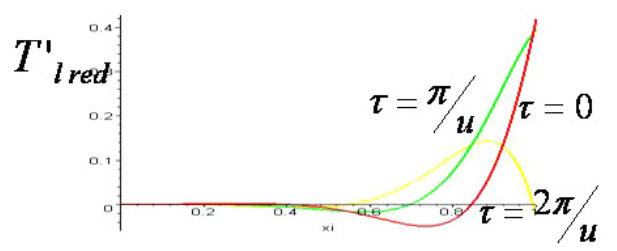

(f)

Fig. 6. Reduced temperature perturbation inside the pastille: browsing in time over semi period. (a) Isothermal bottom, $\theta=5$, $u=15$. (b) Adiabatic bottom, $\theta=5, u=15$. (c) Isothermal bottom, $\theta=5, u=150$. (d) Adiabatic bottom, $\theta=5, u=150$. (e) Isothermal bottom, $\theta=5, u=1500$. (f) Adiabatic bottom, $\theta=5, u=1500$.

when $u$ takes the values $15 \times 10^{3}, 15 \times 10^{5}$, and $15 \times 10^{6}$, respectively and then $\Delta_{l}$ is reduced even for a comparatively high value of $\theta(\theta=50$ for example). For the last value of $u\left(u=15 \times 10^{6}\right)$, the penetration depth $\Delta_{l}$ of the wave is nearly zero for $\theta=50$.

In the adiabatic bottom regime, the penetration depth $\Delta_{l}$ of the wave increases with $\theta$ for a fixed frequency $u$. Moreover, the oscillation amplitude remains high for $\xi$ values near zero for a large value of $\theta$ combined with a small value of $u$. A typical case is the one with $u$ fixed at 15 and $\theta$ at 50. In this case $\Delta_{l}=1$ and the amplitude of the trio of curves remains uniformly close to the one of the values at the evaporating pastille surface. One may conjecture that in this case the perturbation of the reduced temperature at the surface propagates instantly with the same amplitude toward the bottom of the pastille. As in the isothermal bottom regime, the increase of $u$ at a fixed $\theta$ tends to reduce the penetration depth $\Delta_{l}$ of the perturbation (Fig. 6). Remarkably, when $\theta=50$ and $u=15 \times 10^{6}$ the penetration depth $\Delta_{l}$ of the wave is nearly zero.

\subsection{Analysis of results}

The curves for the isothermal and adiabatic bottom regimes are similar or even identical for $(\theta=0.1, u \geq 15)$; $(\theta=5, u=1500)$; and $(\theta=50, u \geq 15000)$. Accordingly, the $3 \mathrm{D}$ graphs in Figures $7 \mathrm{e}$ and $\mathrm{f}$ seem to be identical for isothermal and adiabatic bottom regimes. We may estimate for the ratio $\frac{u}{\theta}$ the upper bound value $R_{0}$ starting from which the propagation of the perturbation of the reduced temperature $T_{\text {lred }}^{\prime}$ in the pastille is identical for isothermal or adiabatic bottom cases. This value turns out to be about 100 .

Below we describe an attempt to evaluate for the ratio $\frac{u}{\theta}$, we attempt the lower bound value $r_{0}$ below which notable differences in the amplitude of the perturbation propagation along the abscissa $\xi$ appear between the isothermal and adiabatic bottom regimes. We consider for comparisons a sample of 56 different values of this ratio for both isothermal and adiabatic bottom regimes. For the values of $u>u^{*}$ with $u^{*} \approx 15$, notable differences between the two cases appear for $\frac{u}{\theta}<\frac{u^{*}}{\theta^{*}} \approx 3$, that is for $\omega \widetilde{\tau}_{\mathrm{T}}<1$. The inequality $\omega \widetilde{\tau}_{\mathrm{T}}<1$ signifies that the thermal diffusion time $\tilde{\tau}_{\mathrm{T}}$ is smaller than the period of the harmonic perturbation. The heat transfer by diffusion from the evaporating surface reaches the bottom of the pastille before the thermal perturbation wave. This fact induces a notable difference in the thermal wave propagation between the isothermal and adiabatic bottoms cases, since the boundary conditions relative to the conduction at the bottom of the pastille are kept different.

For the values of $u \leq u^{*}$, that is with $\omega \bar{\tau}_{v} \leq \frac{u^{*}}{3}\left(\omega \bar{\tau}_{v} \leq\right.$ $5)$, the differences between the two cases seem to occur for $\theta^{*} \leq \theta$ with $\theta^{*} \approx 5$, and this happens independently of the value of the ratio $\frac{u}{\theta}$. For frequency value $u$ smaller 


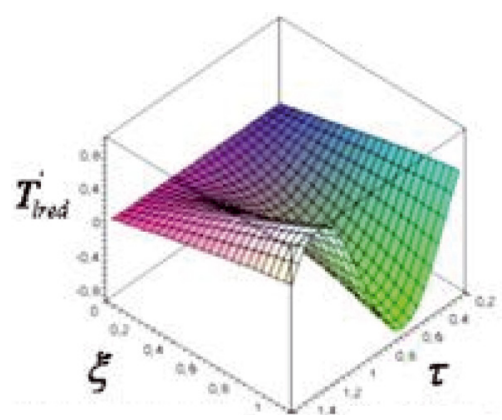

(a)

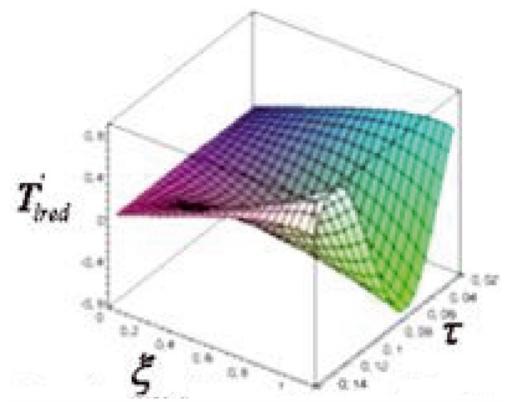

(c)

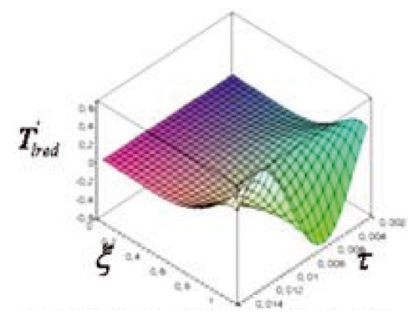

(e)

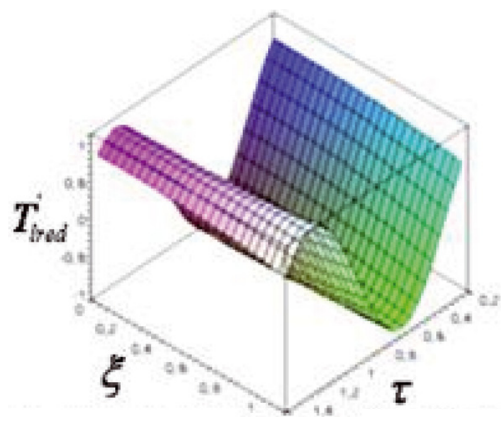

(b)

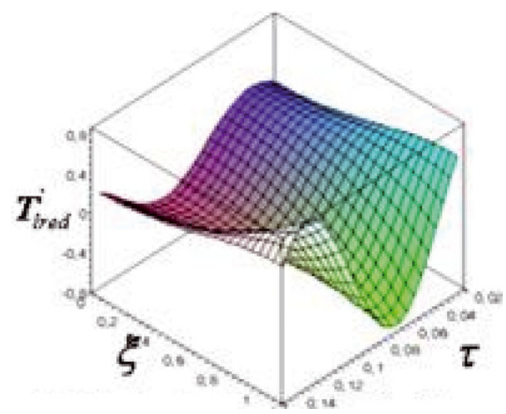

(d)

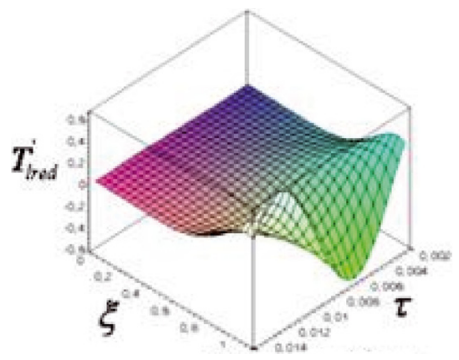

(f)

Fig. 7. Differences between reduced temperature perturbation $T_{\text {lred }}^{\prime}$ propagation inside the pastille (3D view) according to boundary condition at $x=0$. Abscissa values are in the range $\xi=0, \ldots, 1$ and time $\tau=\frac{\pi}{u}, \cdots \frac{7 \pi}{u} ; T_{\text {lred }}^{\prime}$ varies in the range $-1, \ldots, 1$. Variables $T_{\mathrm{lred}}^{\prime}, \xi$ and $\tau$ are defined in Section 5.1. (a) Isothermal bottom, $\theta=10, u=15$. (b) Adiabatic bottom, $\theta=10, u=15$. (c) Isothermal bottom, $\theta=10, u=150$. (d) Adiabatic bottom, $\theta=10, u=150$. (e) Isothermal bottom, $\theta=10$, $u=1500$. (f) Adiabatic bottom, $\theta=10, u=1500$.

than $u^{*} \approx 15$, the residence time $\bar{\tau}_{v}$ of the injected liquid is less than the period of the harmonic perturbation. The inequality $\theta^{*} \leq \theta$ signifies, on the other hand, that the thermal diffusion time $\tilde{\tau}_{\mathrm{T}}$ is also less than the residence time $\bar{\tau}_{v}$. The thermal perturbation propagation extends toward the bottom of the pastille where the boundary condition is kept either isothermal or adiabatic.

The differences between the isothermal and adiabatic bottom are then manifested in the following range of $u$ values:

$$
\left\{\begin{array}{l}
u \leq u^{*} \\
\theta^{*} \leq \theta
\end{array}\right.
$$

or

$$
\left\{\begin{array}{l}
u^{*}<u \\
\frac{u}{\theta}<\frac{u^{*}}{\theta^{*}}
\end{array}\right.
$$

It is better to have only one inequality to summarize these conditions. The use of the absolute value function for this purpose is similar to the common usage of the Heaviside function. The limit with the negligible differences region may be characterized by a formula delimiting the area of significant differences:

$$
2\left(\frac{u^{*}}{\theta^{*}}\right) \theta \geq u+u^{*}+\left|u-u^{*}\right|
$$

In effect, the inequality (19) provides for $u^{*}<u$ : $\left|u-u^{*}\right|=u-u^{*}$ which implies $\frac{u}{\theta}<\frac{u^{*}}{\theta^{*}}$. For $u^{*} \geq u$ : $\left|u-u^{*}\right|=u^{*}-u$ which implies $\theta^{*} \leqslant \theta$. Therefore $r_{0}$ can be approximately estimated to be about $\frac{u^{*}}{\theta^{*}}=3$. One may notice that $\bar{\tau}_{v}$ do not intervene in these relations. Only $\tilde{\tau}_{\mathrm{T}}$ and $\omega=\frac{2 \pi}{\Gamma}$ intervene, $\Gamma$ being the period of the harmonic perturbation. For a given $\bar{\tau}_{v}$ the later inequality becomes 


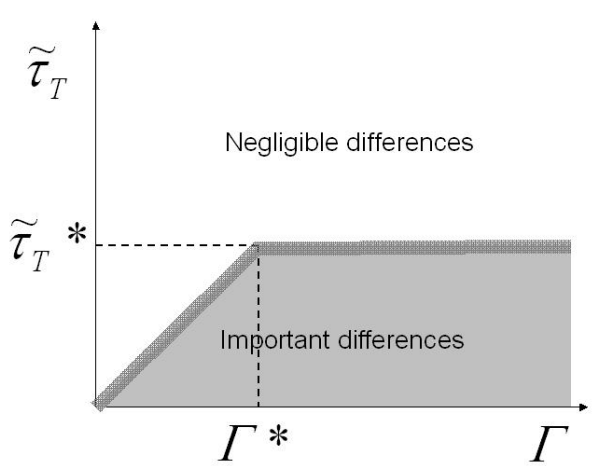

Fig. 8. Differences between the temperature fields of liquid for the two conditions in $x=0$ : isothermal (imposed temperature) and adiabatic (zero thermal conduction).

$$
2 \frac{\Gamma}{\Gamma^{*}} \geq \frac{\tilde{\tau}_{\mathrm{T}}}{\tilde{\tau}_{\mathrm{T}}^{*}}\left(1+\frac{\Gamma}{\Gamma^{*}}+\left|1-\frac{\Gamma}{\Gamma^{*}}\right|\right)
$$

The two areas, of negligible differences (in white) and important differences (in grey) between the isothermal and adiabatic bottom cases, are illustrated in Figure 8.

In conclusion, we understand that important differences between the two regimes occur if the thermal perturbation reaches the bottom of the pastille after the heat diffusion transfer is realized inside the pastille. This is possible if the thermal diffusion time $\tilde{\tau}_{\mathrm{T}}$ is roughly less than one oscillation period and than the residence time $\bar{\tau}_{v}$. The calculations conducted for the pastille with $A=10$ and $B=100$ give $\theta^{*} \approx 5$ and $u^{*} \approx 15$.

\section{Conclusions}

We studied analytically the dynamic response of an evaporating droplet subjected to acoustic oscillation using a "pastille-shaped" model. Like other authors, we approached this problem by considering a continuously fed drop at rest. Our model does not consider the spherical geometry of the drop, but the resulting simplification has better taken into account the convection effect inside the drop and the boundary conditions controlling the vaporization process. This model permits to broaden the range of parameters of the problem and therefore seems to be more realistic.

Two thermal forcing types were considered: constant temperature (isothermal case) and zero temperature gradient (adiabatic case). Important differences were observed between the cases analyzed, but similarities were also observed. It was found that if the response factor has positive values, pointing to instability, its maximum is always reached when the residence time is of the same order of magnitude as the period of the harmonic disturbance. Likewise, the temperature disturbance yields a wave penetration depth inside the pastille. If this depth is smaller than the pastille thickness, the thermal forcing mechanism has little influence on the temperature perturbation inside the pastille. The isothermal injection leads to lower response factors and may even suppress the instability, unlike the adiabatic injection which maintains a zone of instability. The temperature profiles in the liquid are very different in both cases when the thermal diffusion time is smaller than the period of the harmonic oscillation and than the residence time. Thermal disturbances are more important in the case of an adiabatic feeding. This study and its results may be relevant to the development of numerical codes for calculating two-phase flows with a consideration of thermal structures inside the droplets. The analytical solutions can serve as references to validate the codes and the physical results obtained may help to interpret the observed behavior since the lifetime of a vaporizing droplet without feeding can be assimilated to the residence time for a supplied droplet.

The application of the frequency response characteristics of the vaporization process to combustion chamber design may be envisioned in several ways. First, according to the influence of the boundary conditions controlling the vaporization process, the isothermal feeding can better contribute to design stable combustion chamber (with the response factor less than zero) than the adiabatic one. Since the thermal convection have significant reduction effect on instability due to acoustic oscillations, arbitration may be made between the speed of the injection rate and the size (length or radius) of the combustion chamber. We have also shown that the residence time, the thermal diffusion time, and the period of the harmonic perturbation do intervene strongly in the behaviour of the process. It is found that the residence time depends on the size of the drop, the thermal diffusion time depends on the propellant diffusivity, and the period of the oscillation can be related to the shape of the combustion chamber. The judicious choice of these parameter values in agreement with the main results of this paper may permit to obtain more stability in the combustion chamber.

\section{References}

[1] R. Prud'homme, M. Habiballah, L. Matuszewski, Y. Mauriot, A. Nicole, Theoretical analysis of dynamic response of a vaporizing droplet to a acoustic oscillation, J. Propuls. Power (0748-4658) 26 (2010) 1

[2] R. Bhatia, W.A. Sirignano, One-dimensional analysis of liquid-fuel combustion instability, J. Propulsion 7 (1991) 953-961

[3] F. Culick, V. Yang, Overview of combustion instabilities in liquid-propellant rocket engines, Liquid Rocket Engine Combustion Instability AIAA 169 (1995) 3-37

[4] J.-P. Delplanque, W.A. Sirignano, Transcritical liquid oxygen droplet vaporization: effect on rocket combustion instability, Atomization and sprays 4 (1996) 325-349

[5] M. DiCicco, J. Buckmaster, Acoustic instabilities driven by slip between a condensed phase and the gas-phase in combustion systems, 32nd AIAA Aerospace Sciences Meeting and Exhibit, Paper AIAA 94-0103, Reno, NV (USA), 1994, pp. 10-13

[6] I. Dubois, M. Habiballah, R. Lecourt, Numerical analysis of liquid rocket engine combustion instability, 33rd AIAA Aerospace Sciences Meeting and Exhibit, Paper AIAA95-0607, Reno, NV (USA), 1995, pp. 9-12 
[7] A. Duvur, C.H. Chiang, W.A. Sirignano, Oscillatory fuel droplet vaporization: driving mechanism for combustion instability, J. Propuls. Power 12 (1996) 358-365

[8] D.T. Harrje, F.H. Reardon, Liquid propellant rocket combustion instability, NASA SP-194, 1972

[9] M.F. Heidmann, P.R. Wieber, Analysis of frequency response characteristics of propellant vaporisation, NASA Technical Note D-3749, 1966

[10] M.F. Heidmann, Frequency response of a vaporization process to distorted acoustic disturbances, NASA Technical Note D-6806, 1972

[11] R. Prud'homme, Evaporation et combustion de gouttes dans les moteurs, Editions Techniques de l'Ingénieur, Traité de Mécanique, BM 2 521, 2009, p. 22

[12] R. Prud'homme, Flows of reactive fluids, Book Series: Fluid Mechanics and Its applications, Springer, 2010, Vol. 94

[13] R. Prudhomme, M. Habiballah, A. Nicole, Y. Mauriot, Instabilités liées au phénomène d'évaporation: Réponse dynamique d'une goutte à un champ acoustique, $17^{\mathrm{e}}$ Congrès Français de Mécanique, Troyes, 2005

[14] S.S. Sazhin, Advanced models of fuel droplet heating and evaporation, Progr. Energ. Combust. Sci. 32 (2006) 162214
[15] L. Rayleigh, The theory of sound, Macmillan, 1945

[16] S.K. Aggarwal, A.Y. Tong, W.A. Sirignano, A comparison of vaporization models in spray calculations, AIAA J. 22 (1984) 1448-1457

[17] R. Prudhomme, M. Habiballah, Évaporation et combustion de gouttes: revue des hypothèses et des principaux résultats de l'analyse quasi-stationnaire, Rapport Technique ONERA N RT 1/05 424, 2001

[18] C.K. Law, Recent advances in droplet vaporization and combustion, Proc. Energy Combustion Science 8 (1982) $171-201$

[19] W.A. Sirignano, J.-P. Delplanque, C.H. Chiang, R. Bhatia, Liquid-propellant droplet vaporization: a rate controlling process for combustion instability, in Liquid rocket engine combustion instability, edited by $\mathrm{V}$. Yang, W.E. Anderson, Progress in Astronautics and Aeronautics 169 (1994), Publ. AIAA

[20] M. De Benedictis, Instabilités couplées haute fréquence dans les moteurs-fusées à ergols liquides : étude du couplage chambre de combustion/système d'alimentation, Thèse, Université de Poitiers, 2007

[21] V. Yang and W. Anderson, Liquid propellant rocket combustion instability, Progress in astronautics and aeronautics, 169, 1995 